\title{
REBECGA Registry: A Multicenter Study for the Reduction of Maternal Mortality Due to Heart Diseases Manifested During Pregnancy
}

\author{
Karyne Pollo de Souza ${ }^{(\mathbb{6})}$ and Christianne Brêtas Vieira Scaramello ${ }^{(\mathbb{b}}$ \\ Universidade Federal Fluminense - Instituto Biomédico, Niterói, RJ - Brazil \\ Editorial referring to the article: The REBECGA Brazilian Registry of Pregnancy and Heart Disease: Rationale and Design
}

According to the literature, although the prevalence of heart disease in pregnancy varies considerably among countries, cardiovascular disease is responsible for $1 \%$ to $4 \%$ of complicated pregnancies in industrialized countries. This pattern is related to cardiovascular risk factors, such as smoking, diabetes, obesity, and hypertension, especially in low- and middle-income countries, as well as late motherhood and the presence of congenital heart disease, mainly in high-income countries. ${ }^{1,2}$

Improved diagnostic methods and therapeutic alternatives have encouraged maternity by promoting safer pregnancies. However, heart disease is still the leading non-obstetric cause of maternal mortality worldwide. Although the rate of maternal mortality has decreased in Brazil over the last three decades, it continues to be higher than estimated for this millennium. ${ }^{3}$

As noticed throughout the world, the percentage of congenital heart disease during pregnancy has risen in Brazil over the past 50 years due to improved surgical and late postoperative treatment that allowed an increased number of children with congenital heart disease to reach childbearing age. The well-accepted World Health Organization classification of the risk stratification model for pregnancy in patients with congenital heart disease considers this condition as risk III, meaning medical advice against pregnancy. ${ }^{4}$

Pregnancy leads to intrinsic hormonal stimulation in the organism and physiological changes of the cardiovascular system, which are necessary for adequate pregnancy development. The consequent hemodynamic

\section{Keywords}

Heart Diseases; Pregnant Women; Maternal Mortality; Multicenter Study. overload may reveal previous heart diseases or aggravate the functional state of underlying heart disease. Thus, it may increase the risk of adverse maternal cardiac, obstetric, fetal, and neonatal outcomes. ${ }^{5}$

The quality of healthcare provided to women during prenatal care reflects the population's living conditions, and a country's maternal mortality rate is one of the most sensitive indicators. ${ }^{3}$ The coexistence of cardiac disease and pregnancy presents challenges across the spectrum of prevention, diagnosis, treatment, and health service delivery that are unique for both mother and baby. ${ }^{5}$

In this context, the proposal of Avila et al. ${ }^{6}$, encompassing the development of a multicenter Brazilian registry of pregnant women with heart disease (REBECGA Registry) with the aim of contributing to planning preventive measures, fundraising for improvement of prenatal care for high-risk pregnancies, and reducing immediate and late maternal mortality due to heart disease is beyond interesting; it is necessary.

Avila et al. ${ }^{6}$ intend to study the causes of immediate and late maternal mortality, ascertaining the prevalence of heart disease in the country's macroregions. To achieve this purpose, they will conduct an observational study, encompassing retrospective (data from medical records between 2017 and 2020) and prospective (data from faceto-face consultations between 2020 and 2026) analysis of clinical and obstetric evolution of pregnant women with heart disease, with one year of follow-up after delivery.

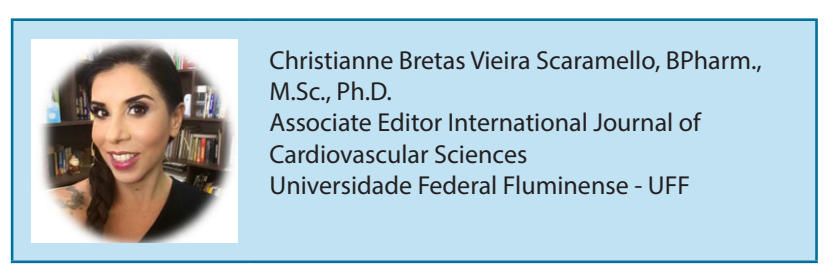

Mailing Address: Christianne Brêtas Vieira Scaramello

Instituto Biomédico - Universidade Federal Fluminense - Rua Prof. Hernani Mello, 101. Postal Code: 24210-130, São Domingos, Niterói, RJ - Brazil E-mail: chrisbretas@gmail.com 
The project will be conducted in public and private hospitals with compatible infrastructure, a responsible researcher, and approval by the respective Ethics and Research Committees. ${ }^{6}$

An Investigation Committee, formed by members from the Department of Women's Cardiology of the Brazilian Society of Cardiology, will select the participating centers and coordinate the platform of the data record. The responsible researcher from each center will select patients according to the defined inclusion and exclusion criteria, manage incomplete data, insert data in the Research Electronic Data Capture (REDCap) platform, and participate in the Committee's meetings to contribute to study progress. ${ }^{6}$

In addition to the inclusion and exclusion criteria, the variables to be analyzed, outcomes, prospective sample size, and statistical analysis have been well defined in the proposal. Furthermore, data quality management and assurance have also been described. Thus, the proposal seems to be well outlined, presenting few specific limitations, which the authors have already mentioned. It is worth noting that the project has been approved by the Ethics and Research Committee of Hospital of the

\section{References}

1. Guimarães T, Magalhães A, Veiga A, Fiuza M, Ávila W, Pinto FJ. Heart Disease and Pregnancy: State of the Art. Rev Port Cardiol. 2019;38(5):37383. doi: 10.1016/j.repc.2018.05.013.

2. Testa CB, Borges VTM, Bortolotto MRFL. Pregnancy in Patients With Heart Disease. Rev Med. 2018; 97(2):177-86. doi: 10.11606/issn.1679-9836. v97i2p177-186.

3. Avila WS, Alexandre ERG, Castro ML, Lucena AJG, Marques-Santo C, Freire CMV, et. al. Brazilian Cardiology Society Statement for Management of Pregnancy and Family Planning in Women with Heart Disease - 2020. Arq Bras Cardiol. 2020; 114(5):849-942. doi: 10.36660/ abc. 20200406 .

4. Avila WS, Ribeiro VM, Rossi EG, Binotto MA, Bortolotto MR, Testa C, Francisco R, et al. Pregnancy in Women with Complex Congenital Heart Disease. A Constant Challenge Arq. Bras. Cardiol. 2019;113(6):1062-9. doi: 10.5935/abc. 20190197.

5. Parsonage WA, Zentner D, Lust K, Kane SC, Sullivan EA. Heart Disease and Pregnancy: The Need for a Twenty-First Century Approach to Care.... Heart Lung Circ. 2021;30(1):45-51. doi: 10.1016/j.hlc.2020.06.021.
Clinics, Faculty of Medicine, University of São Paulo, which is the coordinating center of the study. ${ }^{6}$

A similar study was conducted by Roos-Hesselinket et al. ${ }^{7}$ They observed that patients with cardiomyopathy and pulmonary arterial hypertension are high risk, while relatively good outcomes are expected for women with congenital heart disease. They stated that pregnancy should only be discouraged to women with very high risks. They also quoted some pre-pregnancy predictors for mortality and/or heart failure, such as NYHA class $>$ II, systemic ventricular ejection fraction $<40 \%$, signs of heart failure, and the use of anticoagulants. Roos-Hesselinket et al. ${ }^{7}$ concluded that, after 2010, maternal mortality and/ or heart failure rates decreased, particularly in emerging countries, despite the increased number of high-risk patients, highlighting the importance of further studies to assess the optimal management of these patients.

Brazil is a continent-sized country. The literature discusses that continental dimensions, heterogeneity, and wide local diversity have to be considered to define public policy and healthcare, ${ }^{8-10}$ making the proposal by Avila et al. ${ }^{6}$ even more remarkable, given that they intend to analyze the macroregions of the country.

6. Avila W, Rivera MAM, Marques-Santos C, Rivera IR, Costa MENC, Lucena AJG, et al. The REBECGA Brazilian Registry of Pregnancy and Heart Disease: Rationale and Design. Int J Cardiovasc Sci. 2021; 34(4):452458. doi: https://doi.org/10.36660/ijcs.20200419.

7. Roos-Hesselink J, Baris L, Johnson M, Backer J, Otto C, Marelli A, et al. Pregnancy Outcomes in Women with Cardiovascular Disease: Evolving Trends Over 10 years in the ESC Registry Of Pregnancy And Cardiac disease (ROPAC). Eur Heart J. 2019;40(47):3848-55. doi: 10.1093/eurheartj/ ehz136.

8. Cecilio LCO, Reis AACD. Notes on Persistent Challenges for Basic Health Care in Brazil. Cad Saude Publica. 2018;34(8):e00056917. doi: 10.1590/0102-311X00056917.

9. Koga D. Experiencing territories in a continental country. Serv Soc Saude. 2015;14(1):9-26.

10. Busingye D, Arabshahi S, Subasinghe AK, Evans RG, Riddell MA, Thrift AG. Do the Socioeconomic and Hypertension Gradients in Rural Populations of Low- and Middle-Income Countries Differ by Geographical Region? A Systematic Review and Meta-Analysis. Int J Epidemiol. 2014;43(5):1563-77. doi: 10.1093/ije/dyu112. 